\title{
GENERALIZED TWO POINT BOUNDARY VALUE PROBLEMS. EXISTENCE AND UNIQUENESS ${ }^{1}$
}

\author{
K.N. MURTY ${ }^{2}$ \\ Department of Applied Mathematics \\ Florida Institute of Technology \\ $150 \mathrm{~W}$. University Blvd. \\ Melbourne, Florida 32901-6988, USA \\ S. SIVASUNDARAM \\ Department of Mathematics \\ Embry-Riddle Aeronautical University \\ Daytona Beach, Florida 32114, USA
}

\begin{abstract}
An algorithm is presented for finding the pseudo-inverse of a rectangular matrix. Using this algorithm as a tool, existence and uniqueness of solutions to two point boundary value problems associated with general first order matrix differential equations are established.
\end{abstract}

Key words: Fundamental matrix, characteristic matrix, generalized inverse, Green's matrix.

AMS (MOS) subject classification(s): $\quad 34 \mathrm{~B} 10,34 \mathrm{~B} 27,15 \mathrm{~A} 09$.

\section{INTRODUCTION}

In this paper we consider the general first order matrix differential equation of the

form

$$
L y \equiv P(t) y^{\prime}+Q(t) y=f(t), a \leq t \leq b,
$$

where $P, Q \in\left[L_{p}(a, b)\right]^{m \times n}$ and $f \in\left[L_{p}(a, b)\right]^{n}$ for some $p$ satisfying the condition $1 \leq p \leq \infty$. We seek a solution of (1.1) satisfying the boundary condition

$$
B y \equiv M y(a)+N y(b)=d .
$$

(In case of multipoint $B y \equiv \sum_{i=1}^{n} M_{i} y\left(t_{i}\right)=d, a=t_{1}<t_{2}<\ldots<t_{n}=b$ and the matrices $\left.M_{i} \in R^{n \times m}\right)$, where $M, N \in R^{n \times m}$ and $d \in R^{n}$.

\footnotetext{
${ }^{1}$ Received: April, 1991. Revised: July, 1991.

${ }^{2}$ Visiting from Andra University, Department of Applied Mathematics, Visakapatnam (AP), INDIA.
} 
In the case $m=n$, the boundary value problem (1.1) and (1.2) is said to be invertible if the homogeneous BVP (1.1) and (1.2) with $f=0$ and $d=0$ has only the trivial solution $y=0$. Equivalently, the BVP (1.1), (1.2) has a unique solution $y$ if and only if the characteristic matrix $D=M Y(a)+N Y(b)$ is non-singular (where $Y$ is a fundamental matrix of $L y=0)$. In this case one can formally write the solution as

$$
y(t)=Y(t) D^{-1} d+\int_{a}^{b} G(t, s) f(s) d s .
$$

Here $G$ is the Green's matrix for the homogeneous BVP and is given by

$$
G(t, s)=\left\{\begin{array}{l}
Y(t) D^{-1} M Y(a) Y^{-1}(s) P^{-1}(s), a \leq s \leq t \leq b \\
-Y(t) D^{-1} N Y(b) Y^{-1}(s) P^{-1}(s), a \leq t \leq s \leq b .
\end{array}\right.
$$

In the non-invertible case we develop a method to compute the generalized inverse of a matrix and using this technique we establish a unique solution of the two point BVP (1.1) and (1.2). This technique is substantially new and includes the existing results as a particular case.

\section{PRELIMINARIES}

Suppose that $A$ is $(m \times n)$ matrix of rank $r$. We partition $A$ in the following manner.

$$
A=\left(\begin{array}{ll}
A_{11} & A_{12} \\
A_{21} & A_{22}
\end{array}\right) \text {. }
$$

Here $A_{11}$ is an $(r \times r)$ matrix, $A_{12}$ is an $(r \times(n-r))$ matrix, $A_{21}$ is an $((m-r) \times r)$ matrix and $A_{22}$ is an $((m-r) \times(n-r))$ matrix. Since the rank of $A$ is $r$, the last $m-r$ rows of $A$ are linear combinations of the first $r$ rows. Therefore an $((m-r) \times r)$ matrix $K$ exists such that

$$
A_{21}=K A_{11} \text { and } A_{22}=K A_{12}
$$

Similarly, an $(r \times(n-r))$ matrix $L$ exists such that

$$
A_{12}=A_{11} L \text { and } A_{22}=A_{21} L .
$$

From (2.2) and (2.3), we get $A_{22}=K A_{11} L$. Hence, $A$ can be written as

$$
A=\left(\begin{array}{cc}
A_{11} & A_{11} L \\
K A_{11} & K A_{11} L
\end{array}\right)=\left(\begin{array}{c}
I \\
K
\end{array}\right) A_{11}\left(\begin{array}{ll}
I & L
\end{array}\right) .
$$

We make use of the following expression given in [2] for the generalized inverse $A^{+}$of $A$ and if we write $A$ in the form $A=B C$, where $B$ and $C$ are both of rank $r$, then 


$$
A^{+}=C^{T}\left(C C^{T}\right)^{-1}\left(B^{T} B\right)^{-1} B^{T}
$$

From (2.4), we can choose $B$ and $C$ as

$$
B=\left(\begin{array}{c}
I \\
K
\end{array}\right) A_{11} \text { and } C=\left(\begin{array}{ll}
I & L
\end{array}\right) .
$$

More specifically, we can associate $A_{11}$ with $C$ instead of $B$. In both cases, (2.5) gives

$$
\begin{aligned}
A^{+} & =\left(\begin{array}{c}
I \\
L^{T}
\end{array}\right)\left(I+L L^{T}\right)^{-1} A_{11}^{-1}\left(I+K^{T} K\right)^{-1}\left(\begin{array}{ll}
I & K^{T}
\end{array}\right) \\
& =\left(\begin{array}{c}
I \\
L^{T}
\end{array}\right)\left(I+K^{T} K\right) A_{11}\left(I+L L^{T}\right)^{-1}\left(\begin{array}{ll}
I & \left.K^{T}\right) .
\end{array}\right.
\end{aligned}
$$

The equation (2.7) involves only one inversion of an $(r \times r)$ matrix. In form (2.6), it may be useful to remember that

$$
\left(I+K^{T} K\right)^{-1}=I-K^{T}\left(I+K K^{T}\right)^{-1} K
$$

since $I+K^{T} K$ is non-singular and in fact it is positive definite,

$$
\begin{gathered}
U^{T}\left(I+K^{T} K\right) U=U^{T} U+(K U)^{T}(K U)>0 \text { if } u \neq 0 \\
\text { and } I+K^{T} K=\left(I K^{T}\right)\left(\begin{array}{c}
I \\
K
\end{array}\right) .
\end{gathered}
$$

By the standard theorem on determinant of the product of rectangular matrices, if $C$ is an $(m \times n)$ matrix $(m>0)$, then $\operatorname{det}\left(C^{T} C\right)=\sum_{K}\left(\operatorname{det} C_{K}\right)^{2}$, where $C_{K}$ is an $(n \times n)$ matrix obtained by selecting any $n$ rows of $A$, and the sum is taken of the square of the determinant overall such matrices.

If we apply this to $(2.8)$, we get

$$
\operatorname{det}\left(I+K^{T} K\right) \geq(\operatorname{det} I)^{2}=1 .
$$

Hence $\left(I+K^{T} K\right)$ is a well conditioned matrix.

A useful method for finding the matrices $K, L$ and $A_{11}^{-1}$ in (2.6) is to just make use of Gauss-Jordan procedure. If we write

$$
\left(\begin{array}{ccc}
A_{11} & A_{12} & I \\
A_{21} & A_{22} & 0
\end{array}\right)
$$


then by using row operations as in Gaussian procedure, the matrix $A_{11}$ can be reduced to the unit matrix of order $I$ and $A_{21}$ can be reduced to zero matrix. The last operation is equivalent to $A_{21}-K A_{11}=0$. The matrix (2.9) is then reduced to

$$
\left(\begin{array}{ccc}
I & A_{11}^{-1} A_{12} & A_{11}^{-1} \\
0 & A_{22}-K A_{12} & -K
\end{array}\right) .
$$

If we use (2.2) and (2.3), the above matrix is equivalent to

$$
\left(\begin{array}{ccc}
I & L & A_{11}^{-1} \\
0 & 0 & -K
\end{array}\right) \text {. }
$$

To illustrate the procedure discussed in this section, we consider the following $(6 \times 4)$ rectangular matrix

$$
A=\left(\begin{array}{cccc}
-1 & 0 & 1 & 2 \\
-1 & 1 & 0 & -1 \\
0 & -1 & 1 & 3 \\
0 & 1 & -1 & -3 \\
1 & -1 & 0 & 1 \\
1 & 0 & -1 & -2
\end{array}\right)
$$

We first reduce this matrix to the row echelon form and from there we see that its rank is 2 . Obviously the $(2 \times 2)$ matrix in left upper of $A$ is non-singular. If we add a $(2 \times 2)$ unit matrix and a $(4 \times 2)$ null matrix to the right of $A$ to form $(2.9)$ and perform elementary row operations to reduce the resulting matrix to the form (2.10), we get

$$
\left(\begin{array}{cccccc}
1 & 0 & -1 & -2 & -1 & 0 \\
0 & 1 & -1 & -3 & -1 & 1 \\
0 & 0 & 0 & 0 & -1 & 1 \\
0 & 0 & 0 & 0 & 1 & -1 \\
0 & 0 & 0 & 0 & 0 & 1 \\
0 & 0 & 0 & 0 & 1 & 0
\end{array}\right) .
$$


We deduce immediately from this

$$
A_{11}^{-1}=\left(\begin{array}{cc}
-1 & 0 \\
-1 & 1
\end{array}\right), K=\left(\begin{array}{cc}
1 & -1 \\
-1 & 1 \\
0 & -1 \\
-1 & 0
\end{array}\right) \text { and } L=\left(\begin{array}{cc}
-1 & -2 \\
-1 & -3
\end{array}\right)
$$

Substituting these forms either in (2.6) or (2.7) yields

$$
A^{+}=\frac{1}{102}\left(\begin{array}{cccccc}
-15 & -18 & 3 & -3 & 18 & 15 \\
8 & 13 & -5 & 5 & -13 & -8 \\
7 & 5 & 2 & -2 & -5 & -7 \\
6 & -3 & 9 & -9 & 3 & -6
\end{array}\right) \text {. }
$$

\section{GENERALIZED TWO-POINT BOUNDARY VALUE PROBLEMS}

We consider in this section the BVP (1.1) and (1.2) and assume that rank of $P(t)$ is $r$. We write $P(t)$ in the form

$$
P(t)=\left(\begin{array}{cc}
P_{11} & P_{12} \\
P_{21} & P_{22}
\end{array}\right)
$$

where $P_{11}$ is $(r \times r), P_{12}$ is $(r \times(n \times r)), P_{21}$ is $((m-r) \times r)$ and $P_{22}$ is $((m-r) \times(n-r))$ and arranged (by interchanging columns and rows necessary) such that $P_{11}$ is non-singular. We apply the technique developed in Section 2, to compute the generalized inverse of $P(t)$ which is given by

$$
P^{+}=\left(\begin{array}{c}
I \\
L^{+}
\end{array}\right)\left(I+L L^{T}\right)^{-1} P_{11}^{-1}\left(I+K^{T} K\right)^{-1}\left(\begin{array}{ll}
I & \left.K^{T}\right) .
\end{array}\right.
$$

We further assume that $Q$ is in the column space of $P(t)$. By this we mean that there exists an $(n \times n)$ matrix $R$ such that

$$
Q=P R \text { and } P+P B=B
$$


for any $B$ for which the matrix $P^{+} P B$ is defined with these conditions. The operator $L Y=0$ is equivalent to

$$
y^{\prime}=A y
$$

where $A=-\left(P^{+} Q\right) \in\left[L_{p}(a, b)\right]^{m \times n}$ and hence a fundamental matrix $Y(t)$ of the system (3.3) exists.

Lemma 3.1: $\quad$ Suppose $Q(t)$ is in the column space of $P$ and $P$ satisfies (3.2). Then, if $Y(t)$ is a fundamental matrix of (3.3) and $\bar{y}(t)$ is a particular solution of (1.1), any solution $y(t)$ of $(1.1)$ is given by

$$
y(t)=\bar{y}(t)+Y(t) C
$$

for some $C \in V_{n}(R)$.

Proof: $\quad$ Noting the fact that $P^{+} P y^{\prime}=y^{\prime}$, one can easily verify that every solution $y(t)$ of (1.1) is of the form given by (3.4).

Lemma 3.2: $\quad$ Let $P$ satisfy condition (3.2). Then a particular solution $\bar{y}(t)$ of (1.1) is of the form

$$
\bar{y}(t)=Y(t) \int_{a}^{t} Y^{-1}(s) P^{+}(s) f(s) d s
$$

where $Y(t)$ is a fundamental matrix of $y^{\prime}=A(t) y$.

Theorem 3.1: If the boundary value problem (1.1), (1.2) with $f=d=0$ has a characteristic matrix $D=M Y(a)+N Y(b)$ of rank $r$, then its index of compatibility is $n-r$.

Proof: $\quad$ If $P^{+}$is the generalized inverse of $P$ given by (3.1) and satisfying (3.2), then any solution of $(3.3)$ is of the form $y(t)+Y(t) C$ for some $C \in V_{n}(R)$. This satisfies the homogeneous end point condition if and only if

$$
D C=0 .
$$

If rank of $D$ is $r$, then the system (3.5) has precisely $(n-r)$ linearly independent solutions, completing the proof of Theorem 3.1 .

Example 3.1: $\quad$ Consider the following BVP:

$$
L y \equiv\left(\begin{array}{ll}
1 & 0 \\
0 & 1 \\
1 & 0
\end{array}\right) y^{\prime}+\left(\begin{array}{cc}
2 & 1 \\
-2 & 0 \\
0 & 2
\end{array}\right) y=\left(\begin{array}{l}
1 \\
1 \\
0
\end{array}\right)
$$




$$
\left(\begin{array}{ll}
1 & 0 \\
0 & 0 \\
0 & 1
\end{array}\right) y(0)+\left(\begin{array}{ll}
1 & 0 \\
0 & 0 \\
0 & 0
\end{array}\right) y(1)=0
$$

The matrix $P$ can be reduced to row echelon form and from that we can see that its rank is 2 . Using the formula (3.1), we get

$$
P^{+}=\left(\begin{array}{ccc}
0 & -1 & 0 \\
2 & 0 & 0
\end{array}\right)
$$

Hence $P+P y^{\prime}+P^{+} Q y=P^{+} f$ is equivalent to

$$
y^{\prime}=\left(\begin{array}{cc}
0 & 1 \\
-2 & 3
\end{array}\right) y+\left(\begin{array}{l}
0 \\
1
\end{array}\right) \text {. }
$$

A fundamental matrix of this system is given by

$$
Y(t)=\left(\begin{array}{cc}
e^{t} & e^{2 t} \\
e^{t} & 2 e^{2 t}
\end{array}\right) .
$$

Substituting the general form of $y(t)=Y(t) C$ in the end point condition, we get $D C=0$ where

$$
D=\left(\begin{array}{cc}
1+e & 1+e^{2} \\
0 & 0 \\
1 & 2
\end{array}\right)
$$

Since $D$ is a $(3 \times 2)$ matrix of rank 2 , the index of capability of the BVP is zero.

Theorem 3.2: $\quad$ Let $P$ satisfy the condition (3.2) and $Y$ be a fundamental matrix of (3.3) and let $D=M Y(a)+N Y(b)$ be a characteristic matrix of rank $r \leq \min (m, n)$. Then the $B V P(1.1),(1.2)$ has a unique solution in the least square sense and is given by

$$
y(t)=\int_{a}^{b} G(t, s) f(s) d s+Y(t) D^{+} \alpha
$$

where $G$ is the Green's matrix for the homogeneous $T P B V P, D^{+}$is the generalized inverse of $D$ and $[M Y(a)+N Y(b)] C+N Y(b) \int_{a}^{b} Y^{-1}(s) P^{+}(s) f(s) d s=\alpha$.

Proof: $\quad$ From Lemma 3.1 and 3.2, it follows that any solution $y(t)$ of $(1.1)$ is of the form

$$
y(t)=Y(t) C+\int_{a}^{t} Y(t) Y^{-1}(s) P^{+}(s) f(s) d s,
$$


where $P^{+}$is the generalized inverse of $P$ given by $(3.1)$, and also $P^{+}$satisfies the condition (3.2).

Now substituting the general form of $y(t)$ in the boundary condition matrix, we get

$$
[M Y(a)+N Y(b)] C+N Y(b) \int_{a}^{b} Y^{-1}(s) P^{+}(s) f(s) d s=\alpha
$$

or

$$
D C=\alpha-N Y(b) \int_{a}^{b} Y^{-1}(s) P^{+}(s) f(s) d s
$$

Since $D$ is an $(m \times n)$ matrix of rank $r$, using the formula similar to (2.6), we get

$$
\left.C=D^{+} p \alpha-N Y(b) \int_{a}^{b} Y^{-1}(s) P^{+} f(s) d s\right]
$$

where $D^{+}$is calculated from $(2.6)$ and $D^{+}$satisfies the condition $D^{+} D C=C$.

Substituting the general form of $C$ in (3.4), we get

$$
\begin{aligned}
y(t)=Y(t) D^{+} & \alpha-Y(t) D^{+} N Y(b) \int_{a}^{b} Y^{-1}(s) P^{+}(s) f(s) d s \\
& +Y(t) \int_{a}^{t} Y^{-1}(s) P^{+}(s) f(s) d s \\
& =\int_{a}^{b} G(t, s) f(s) d s+Y(t) D^{+} \alpha,
\end{aligned}
$$

where

$$
G(t, s)=\left\{\begin{array}{l}
{\left[Y(t) Y^{-1}(s)-Y(t) D^{+} N Y(b) Y^{-1}(s)\right] P^{+}(s), a \leq s<t \leq b} \\
-Y(t) D^{+} N Y(b) Y^{-1}(s) P^{+}(s), a \leq t<s \leq b
\end{array}\right.
$$

It is easy to generalize these results to multipoint BVP's.

The characteristic matrix for the multipoint boundary value problem is defined as

$$
D=\sum_{i=1}^{n} M_{i} Y\left(t_{i}\right)
$$


and if $D^{+}$is the generalized inverse of $D$ given by (3.1) and satisfying (3.2), we have the following analogous result of Theorem 3.2. As the proof is similar to the proof for 3.2 , we omit the proof of this theorem.

Theorem 3.3: $\quad$ Let $Y$ be a fundamental matrix of (3.3) and $D$ be the characteristic matrix of the multipoint $B V P$ with rank $r \leq \min (m, n)$. Then the multipoint $B V P$ has a unique solution given by

$$
y(t)=\int_{a}^{b} G(t, s) f(s) d s+Y(t) D^{-1} \alpha .
$$

Example 3.2: $\quad$ Consider the following TPBVP

$$
\begin{aligned}
& L y \equiv\left(\begin{array}{ll}
1 & 0 \\
0 & 1 \\
0 & 1
\end{array}\right) y^{\prime}+\left(\begin{array}{ll}
0 & 1 \\
1 & 0 \\
0 & 0
\end{array}\right) y=\left(\begin{array}{l}
2 \\
1 \\
1
\end{array}\right) \\
& \left(\begin{array}{ll}
1 & 1 \\
0 & 1 \\
0 & 0
\end{array}\right) y(0)+\left(\begin{array}{cc}
0 & -1 \\
1 & -1 \\
0 & 0
\end{array}\right) y(1)=\left(\begin{array}{l}
0 \\
0 \\
0
\end{array}\right) .
\end{aligned}
$$

Using the formula (3.1) we get,

$$
P^{+}=\left(\begin{array}{lll}
1 & 0 & 0 \\
0 & 1 & 0
\end{array}\right),
$$

and $P^{+}$satisfies condition (3.2). Therefore a fundamental matrix of (3.3) is given by

$$
Y(t)=\left(\begin{array}{cc}
e^{t} & e^{-t} \\
e^{t} & -e^{-t}
\end{array}\right) .
$$

The characteristic matrix $D$ is given by

$$
D=\left(\begin{array}{cc}
2-e & \frac{1}{e} \\
1 & \frac{2-e}{e} \\
0 & 0
\end{array}\right) .
$$

Using the form (3.1), we get 


$$
D^{+}=\left(\begin{array}{cc}
1 & -\frac{1}{e(2-e)} \\
0 & -1
\end{array}\right)\left(\begin{array}{ccc}
1 & 0 & 1 \\
0 & 1 & 0
\end{array}\right)\left(\begin{array}{ccc}
\frac{1}{2-e} & 0 & 0 \\
\frac{-e}{(e-1)(e-3)} & \frac{e(2-e)}{(e-1)(e-3)} & 0 \\
0 & 0 & 1
\end{array}\right)
$$

Substituting these values in (3.5) we get $G(t, s)$.

\section{SUMMARY}

In case if the BVP (1.1) and (1.2) are non invertible an algorithm is presented for finding the pseudo-inverse of a rectangular matrix. Using these pseudo-inverses existence and uniqueness of solutions to two point boundary value problems associated with general first order matrix differential equations are established. Also two examples are given two explained the above mentioned theory.

\section{REFERENCES}

[1] R.H. Cole, "The Theory of Ordinary Differential Equations", Appleton Century Crofts (1968).

[2] T. Greville, "Some applications of the pseudo-inverse of a matrix", SIAM Rev. 2, (1960), pp. 15-22.

[3] A.B. Israel, "On error bound for generalized inverses", SIAM J. Num. Anal. Vol. 3, No. 4 , (1966), pp. 585-592.

[4] C.R. Rao and S.K. Mitra, "Generalized Inverse of Matrices and its Applications", John Wiley and Sons, New York (1971). 


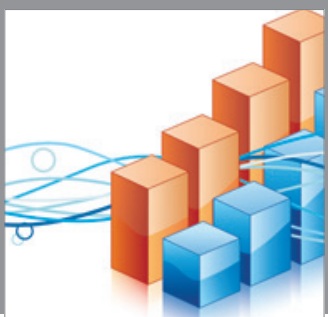

Advances in

Operations Research

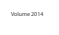

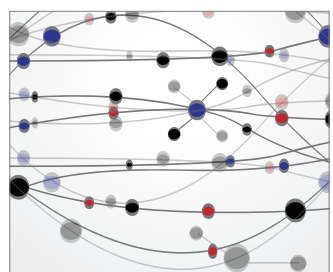

\section{The Scientific} World Journal
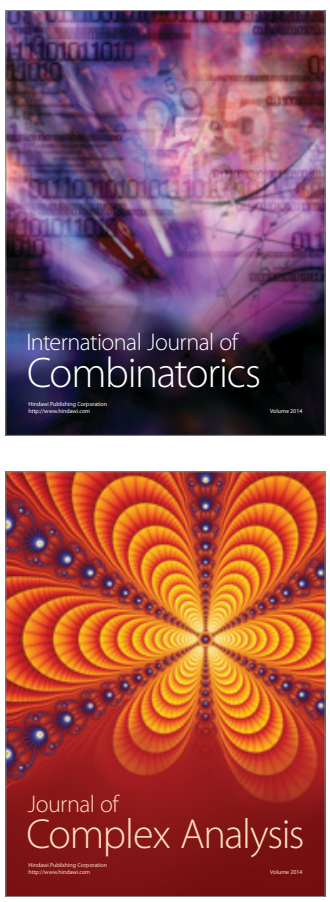

International Journal of

Mathematics and

Mathematical

Sciences
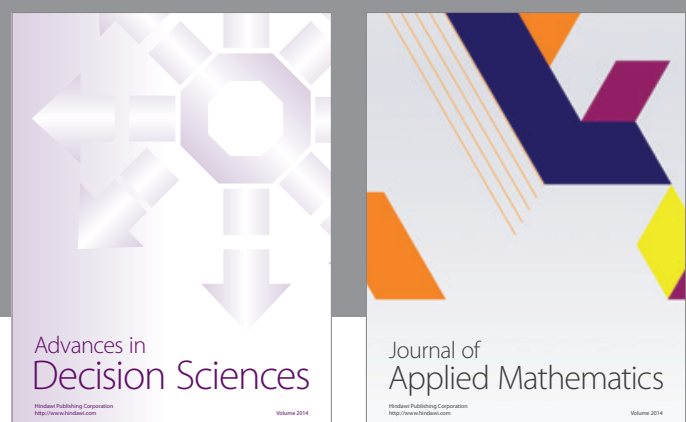

Journal of

Applied Mathematics
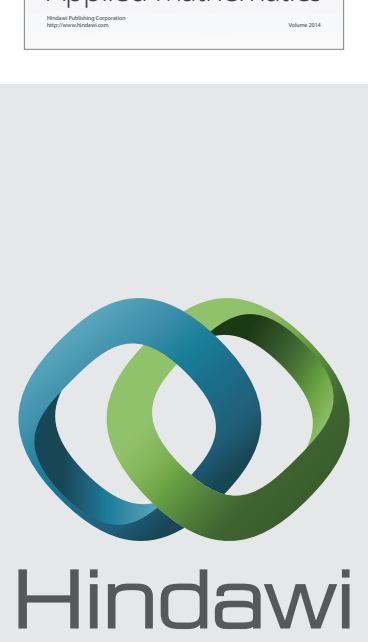

Submit your manuscripts at http://www.hindawi.com
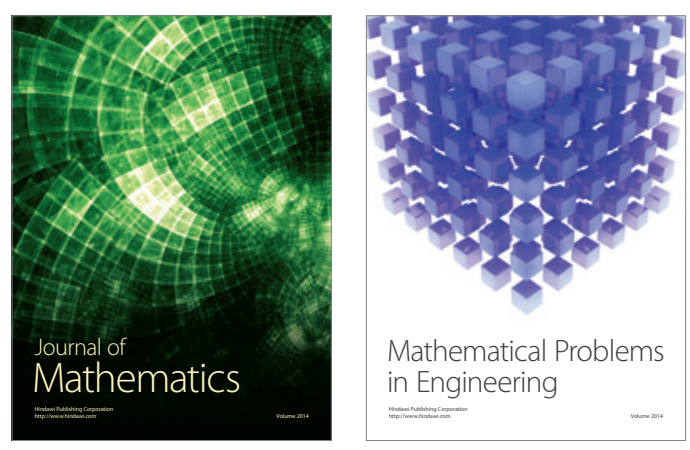

Mathematical Problems in Engineering
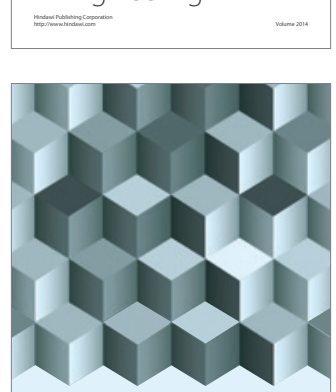

Journal of

Function Spaces
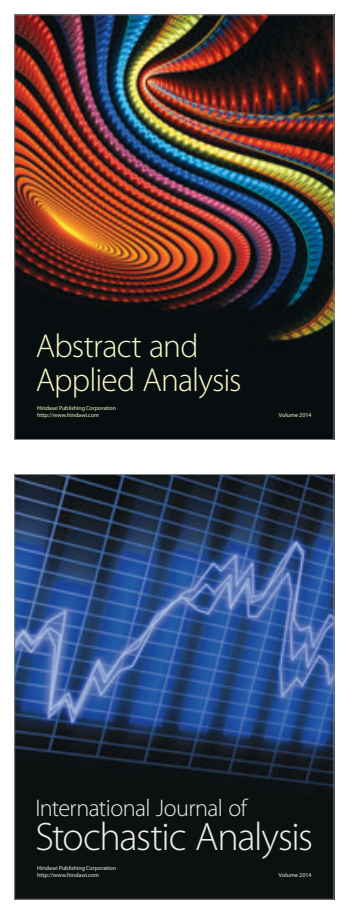

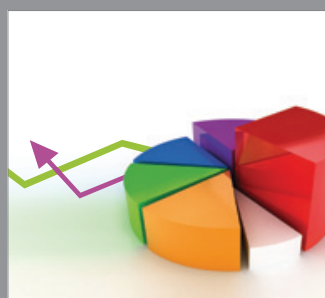

ournal of

Probability and Statistics

Promensencen
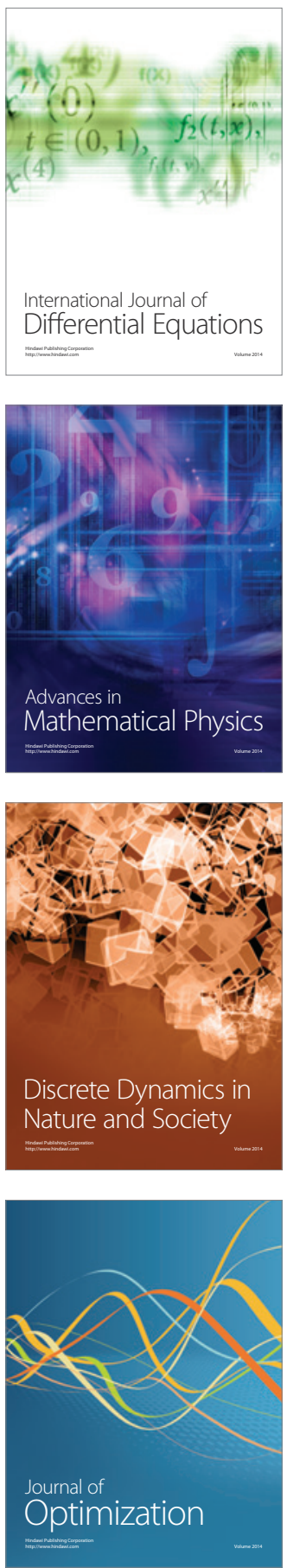